\title{
Análise intraurbana da vulnerabilidade socioambiental no município de Guarulhos no contexto das mudanças climáticas
}

\section{Intra-urban analysis of socio-environmental vulnerability in the municipality of Guarulhos in the context of climate change}

Humberto Prates da Fonseca Alves - Doutor em Ciências Sociais, pela Universidade Estadual de Campinas (Unicamp). Professor da Universidade Federal de São Paulo (Unifesp). E-mail: humbiro@gmail.com

Heber Silveira Rocha-Mestre em Administração Pública e Governo, pela Fundação Getúlio Vargas de São Paulo (FGV). Servidor público da Prefeitura de Guarulhos e docente da Escola de Administração Pública Municipal (ESAP). E-mail: rocha.heber@gmail.com

\begin{abstract}
Resumo
O objetivo do artigo é fazer a identificação e análise espacial de situações de vulnerabilidade socioambiental em escala intraurbana no município de Guarulhos-SP, desenvolvendo uma operacionalização empírica do conceito de vulnerabilidade socioambiental, por meio da integração de indicadores sociodemográficos do Censo Demográfico 2010 com cartografias que representam áreas de risco ambiental. Os resultados revelam que determinadas áreas, espalhadas pelo território do município e onde vivem 119 mil pessoas, possuem alta vulnerabilidade socioambiental e apresentam condições socioeconômicas significativamente piores do que aquelas áreas com baixa e mesmo moderada vulnerabilidade. Portanto, os resultados do artigo podem fornecer subsídios para o planejamento de políticas públicas de mitigação das situações de vulnerabilidade socioambiental e de adaptação às mudanças climáticas, no município de Guarulhos, além de contribuir com o desenvolvimento de indicadores e metodologias para análise de situações de vulnerabilidade às mudanças climáticas, em áreas urbanas e metropolitanas do Brasil.
\end{abstract}

\section{Palavra-chave}

Vulnerabilidade Socioambiental. Indicadores Socioambientais. Mudanças Climáticas. Geoprocessamento. Município de Guarulhos.

\begin{abstract}
The objective of the article is to make the identification and spatial analysis of situations of socio-environmental vulnerability on an intraurban scale in the municipality of GuarulhosSP, developing an empirical operationalization of the concept of socio-environmental vulnerability, through the integration of sociodemographic indicators of the 2010 Demographic Census with cartographies that represent areas of environmental risk. The results reveal that certain areas, spread over the territory of the municipality and where 119 thousand people live, have high socio-environmental vulnerability and have significantly worse socioeconomic conditions than those areas with low and even moderate vulnerability. Therefore, the results of the article can provide subsidies for planning public policies to mitigate situations of socioenvironmental vulnerability and adapt to climate change in the city of Guarulhos, in addition to contributing to the development of indicators and methodologies for analyzing vulnerability situations to climate change in urban and metropolitan areas of Brazil.
\end{abstract}

\section{Keywords}

Socio-environmental Vulnerability. Socioenvironmental Indicators. Climate Change. GIS. Municipality of Guarulhos. 


\section{INTRODUÇÃO}

As áreas urbanas concentram uma proporção grande e crescente das populações mais vulneráveis às mudanças climáticas, tanto nos países do Sul como do Norte. Projeções das Nações Unidas mostram que as populações urbanas dos países em desenvolvimento deverão crescer muito até meados deste século, expondo assim enormes contingentes populacionais a situações de risco ambiental e a desastres naturais. A falta de planejamento adequado para eventos climáticos extremos já provocou inúmeras situações de calamidade em muitas cidades, até mesmo no mundo desenvolvido, como ilustra o caso de Nova Orleans após o furacão Katrina. Portanto, no contexto das mudanças climáticas, nos próximos anos e décadas do século XXI, com cenários de maior frequência e intensidade de eventos climáticos extremos como tempestades, furacões, enchentes e secas, as situações de vulnerabilidade socioambiental tendem a crescer significativamente nas cidades e áreas metropolitanas, principalmente nos países pobres e em desenvolvimento como o Brasil (MARTINE; OJIMA 2013; MOSS et al., 2010; UNFPA, 2007; HUQ et al., 2007).

Entre as consequências da aceleração do processo de urbanização no Brasil, a partir da segunda metade do século XX, pode-se destacar: a formação de regiões metropolitanas, a verticalização e adensamento das áreas já urbanizadas, e a expansão urbana para áreas periféricas. Estudos sobre o processo de urbanização brasileiro mostram que esta expansão urbana para regiões periféricas está ligada à procura por habitação em áreas com baixo preço da terra, provocando um crescimento de assentamentos precários, como favelas e loteamentos irregulares, em locais sem infraestrutura urbana e expostos a risco e degradação ambiental. Como consequência desta dinâmica de urbanização nas áreas periféricas, parte expressiva das áreas urbanas de risco e proteção ambiental, como as margens dos cursos d'água, encontra-se ocupada por habitações precárias de baixa renda, devido à ausência de alternativas habitacionais, tanto por meio do mercado imobiliário privado, como de políticas públicas de habitação (BONDUKI; ROLNIK, 1982; SMOLKA, 1993; MARICATO, 1996, 2003; REIS; TANAKA, 2007; ALVES et al., 2010).

Na Região Metropolitana de São Paulo, desde os anos 1970, tem havido um forte processo de expansão urbana para as áreas periféricas, incorporando um vasto território à mancha urbana metropolitana. Nesse sentido, o crescimento populacional e a periferização têm sido elementos marcantes da Metrópole Paulistana, bem como a pobreza, vulnerabilidade e desigualdade social e 
ambiental. Uma das consequências desses processos é a forte concentração e intensidade de problemas sociais e ambientais em algumas áreas da metrópole, com sobreposição espacial de péssimos indicadores socioeconômicos com riscos de enchentes, deslizamentos de terra, forte poluição ambiental e serviços públicos ineficientes ou inexistentes. Assim, há uma intensa concentração de indicadores negativos em alguns espaços da periferia, sugerindo a presença de "pontos críticos" de vulnerabilidade social e ambiental (MARCONDES, 1999; TORRES et al., 2003; TORRES; ALVES; OLIVEIRA, 2007; TORRES; MARQUES, 2001; ALVES, 2006).

Portanto, esses grandes processos de expansão e degradação das áreas periféricas das metrópoles brasileiras em geral, e da metrópole paulistana em particular, têm revelado um crescente entrelaçamento entre problemas sociais e ambientais, tais como a sobreposição de péssimas condições sociais e sanitárias a riscos e conflitos ambientais (ALVES; TORRES, 2006). Deste modo, um conceito que pode ser usado para analisar estas relações e interações entre as dimensões sociais e ambientais da urbanização é o de vulnerabilidade socioambiental, cuja definição utilizada no presente artigo é a "coexistência, cumulatividade ou sobreposição espacial de situações de pobreza e privação social e de situações de exposição a risco e/ou degradação ambiental" (ALVES, 2013, p. 354-355).

Tendo em vista os elementos expostos acima, o objetivo geral do presente artigo é fazer a identificação e análise espacial de situações de vulnerabilidade socioambiental em escala intraurbana no município de Guarulhos-SP, desenvolvendo uma operacionalização empírica do conceito de vulnerabilidade socioambiental, por meio da integração de indicadores sociodemográficos do Censo 2010 do IBGE (IBGE, 2010a) com cartografias que representam áreas de risco ambiental. A operacionalização do conceito de vulnerabilidade socioambiental será realizada com base em definições que descrevem a vulnerabilidade possuindo duas dimensões (ou dois lados) - 1) exposição ao risco ambiental e 2) suscetibilidade a este risco -, que são expressas no território por meio da sobreposição espacial e cumulatividade de situações de vulnerabilidade social e de situações de exposição a risco ambiental (CHAMBERS, 1989; MOSER, 1998; ALVES, 2006, 2013; DE SHERBININ; SCHILLER; PULSIPHER, 2007).

Deste modo, ao fazer uma análise intraurbana da vulnerabilidade socioambiental de Guarulhos, o presente artigo pode oferecer subsídios para o planejamento de políticas públicas de mitigação das situações de vulnerabilidade socioambiental e de adaptação às mudanças climáticas no município. Além disso, procura-se trazer uma contribuição metodológica para o desenvolvimento de 
indicadores e metodologias de integração de indicadores sociodemográficos e ambientais, por meio do uso de métodos e técnicas de geoprocessamento e de análise espacial de cartografias digitais, para análise de situações de vulnerabilidade às mudanças climáticas, em áreas urbanas e metropolitanas do Brasil.

\section{O CONCEITO DE VULNERABILIDADE: BREVE REVISÃO DA LITERATURA}

As análises empíricas sobre o conceito de vulnerabilidade abrangem um espectro de perspectivas que vão desde abordagens mais sociais até abordagens mais ambientais. Cidade (2013) faz uma revisão bibliográfica dos distintos enfoques sobre a vulnerabilidade, mostrando as dificuldades para construção de uma base conceitual comum. As ciências da natureza teriam como objeto os desastres ambientais, enquanto as ciências sociais, os processos socioeconômicos com efeitos sobre a pobreza. Assim, de um lado a vulnerabilidade social e de outro a vulnerabilidade físico-ambiental correspondem a dois conceitos que representariam duas perspectivas e acepções distintas em relação à vulnerabilidade. Nesse sentido, tem-se buscado perspectivas convergentes, que considerem tanto as dimensões sociais como as físico-ambientais da vulnerabilidade.

Estudos sobre a vulnerabilidade social no Brasil têm sido produzidos com muita frequência por instituições acadêmicas e governamentais nas últimas décadas. $\mathrm{O}$ conceito de vulnerabilidade social tem procurado aperfeiçoar as abordagens tradicionais sobre a pobreza e seus métodos de mensuração, que são baseados exclusivamente no nível de renda monetária e em medidas fixas, como a linha de pobreza (KAZTMAN et al., 1999; ROCHA, 2003).

Já os estudos sobre desastres naturais (natural haz̧ards) e avaliação de risco (risk assessment) deram origem à noção de vulnerabilidade ambiental. Alguns desses estudos descrevem a vulnerabilidade como a interação entre o risco ambiental existente em um determinado local (bazard of place) e as características e o nível de exposição a esse risco da população lá residente. A abordagem físico-ambiental do conceito de vulnerabilidade tem sido desenvolvida principalmente pelos estudos de desastres naturais, com o objetivo de identificar áreas em situação de risco, por meio da elaboração de mapas de suscetibilidades a desastres naturais. Geralmente mais associados com as engenharias, as ciências naturais (Geologia) e a Geografia Física, os estudos sobre a vulnerabilidade físico-ambiental dão mais ênfase às características do meio físico e não se aprofundam nos processos sociais que interagem com esse meio (CUTTER, 1994, 1996; MARANDOLA; HOGAN, 2004; CIDADE, 2013). 
Nos últimos anos, o conceito de vulnerabilidade também tem adquirido grande importância para as comunidades científicas de mudanças climáticas e de mudanças ambientais globais. Com relação às mudanças climáticas, a definição de vulnerabilidade mais utilizada é a do Painel Intergovernamental sobre Mudanças Climáticas (IPCC). Segundo o relatório do IPCC de 2007, vulnerabilidade é a suscetibilidade e incapacidade de um sistema de lidar com os efeitos adversos da mudança climática. Portanto, com base na definição do IPCC, a vulnerabilidade tem três componentes (ou dimensões): grau de exposição ao risco, suscetibilidade ao risco e capacidade de adaptação (ou resiliência) diante da materialização do risco. Nesta perspectiva, as pessoas ou grupos sociais mais vulneráveis seriam aqueles mais expostos a situações de risco ou stress, mais sensíveis a estas situações e com menor capacidade de se recuperar (MOSER, 1998; DE SHERBININ; SCHILLER; PULSIPHER, 2007; IPCC, 2007; OJIMA, 2011).

Segundo Lindoso (2017), pode-se encontrar na literatura diferentes interpretações para a relação entre suscetibilidade, exposição e vulnerabilidade, revelando a existência de uma dicotomia entre os conceitos de vulnerabilidade social e vulnerabilidade físico-ambiental. Para alguns autores, a exposição é parte constitutiva da vulnerabilidade e não pode ser separada da suscetibilidade (O’BRIEN et al., 2004). Porém, existem autores, como Gallopín (2006), que tratam a exposição como elemento independente da suscetibilidade e externo à vulnerabilidade.

Para O’Brien et al. (2007), a vulnerabilidade é um conceito integrador que pode vincular as dimensões social e biofísica da mudança ambiental, e que entrou definitivamente para o "dicionário" dos estudos sobre mudanças climáticas, com cientistas naturais e sociais procurando analisar e medir a vulnerabilidade, seja na perspectiva de regiões, setores, ecossistemas ou grupos sociais. Porém, Adger (2006) adverte que a vulnerabilidade não é facilmente quantificável por meio de uma única métrica. A tradução de um conjunto complexo de parâmetros da vulnerabilidade em uma métrica quantitativa pode encobrir sua complexidade. Apesar disso, nas ciências sociais quantitativas, tem havido um esforço crescente para desenvolver métricas e indicadores de vulnerabilidade, que sejam comparáveis no tempo e no espaço. Estudos que procuram mapear a vulnerabilidade geralmente envolvem comparações de indicadores espaciais em diferentes escalas: global, nacional, regional e local (DOWNING et al., 2001; SAKAI et al., 2017; BALICA et al., 2009; VINCENT, 2004).

Ainda de acordo com Adger (2006), uma boa medida de vulnerabilidade precisa incorporar dimensões temporais e socioespaciais, tendo em vista que muitos fenômenos são transitórios, associados à exposição a riscos específicos, 
e há a necessidade de analisar a distribuição espacial da vulnerabilidade no interior das regiões ou localidades, identificando as características dos grupos sociais mais vulneráveis, e abordando as percepções desses grupos a respeito da vulnerabilidade e do risco.

Graizbord (2013) mapeou áreas de risco ambiental na Região Metropolitana da Cidade do México e identificou populações vulneráveis a eventos extremos de chuva, deslizamentos de terra e ondas de calor, com base em indicadores demográficos e socioeconômicos derivados do censo. $\mathrm{O}$ estudo mostrou que situações de alta vulnerabilidade a desastres naturais afetam $27 \%$ da população da Região Metropolitana da Cidade do México (4,6 milhões de habitantes) e perto de um milhão de domicílios. Assim como no caso da Metrópole de São Paulo, a expansão urbana e as mudanças no uso do solo na Cidade do México têm provocado um aumento da vulnerabilidade das populações mais pobres às mudanças climáticas. Portanto a continuidade dos processos de expansão urbana em áreas metropolitanas dos países em desenvolvimento é motivo de crescente preocupação no contexto das mudanças climáticas (MARTINE; SCHENSUL, 2013; OJIMA; HOGAN, 2009).

Na Metrópole de São Paulo, modelos de projeção da expansão urbana para as próximas décadas revelam um significativo aumento das situações de risco e vulnerabilidade a enchentes, inundações e escorregamentos de terra no contexto das mudanças climáticas. Tais cenários baseiam-se na hipótese de aumento de eventos climáticos extremos e dos riscos ambientais associados, como enchentes e deslizamentos, e de crescimento de assentamentos precários em áreas de risco ambiental, tais como beiras de rios e córregos e terrenos de alta declividade. Neste sentido, situações de vulnerabilidade socioambiental (e climática) estarão cada vez mais presentes na metrópole paulistana nos próximos anos e décadas (NOBRE; YOUNG, 2011; MILANEZ; FONSECA, 2011).

Portanto, tendo em vista esta breve revisão da literatura, é muito importante que haja um esforço para o desenvolvimento de indicadores, metodologias e análises, que permitam identificar, caracterizar e mensurar os diferentes graus de vulnerabilidade às mudanças climáticas nos municípios da Metrópole de São Paulo, como é o caso de Guarulhos, assim como nas demais áreas urbanas e metropolitanas do Brasil, por meio da identificação e caracterização das áreas de maior risco a desastres naturais e dos grupos populacionais mais vulneráveis, complementando a informação qualitativa dos órgãos de defesa civil (BRAGA et al., 2006; CARMO; VALENCIO, 2014). 


\section{METODOLOGIA}

O recorte espacial do estudo é o município de Guarulhos, localizado na Região Metropolitana de São Paulo, a qual abrange 39 municípios (Figura 1). Guarulhos é o segundo município mais populoso do estado de São Paulo, com 1,2 milhões de habitantes no ano de 2010, sendo superado apenas pelo município de São Paulo, com 11,2 milhões de habitantes naquele ano. Entre 2000 e 2010, Guarulhos apresentou uma taxa de crescimento populacional três vezes maior do que a registrada pelo estado de São Paulo. As regiões com maior densidade populacional do município são aquelas de ocupação mais antiga, situadas no centro e seu entorno, seguidas por aquelas que vêm passando por um acelerado processo de adensamento nos últimos anos em função da disponibilidade de áreas livres, nos moldes do referido processo de expansão urbana para regiões periféricas no contexto da metrópole paulistana (IBGE, 2010a).

Figura 1 - Localização do município de Guarulhos na Região Metropolitana de São Paulo

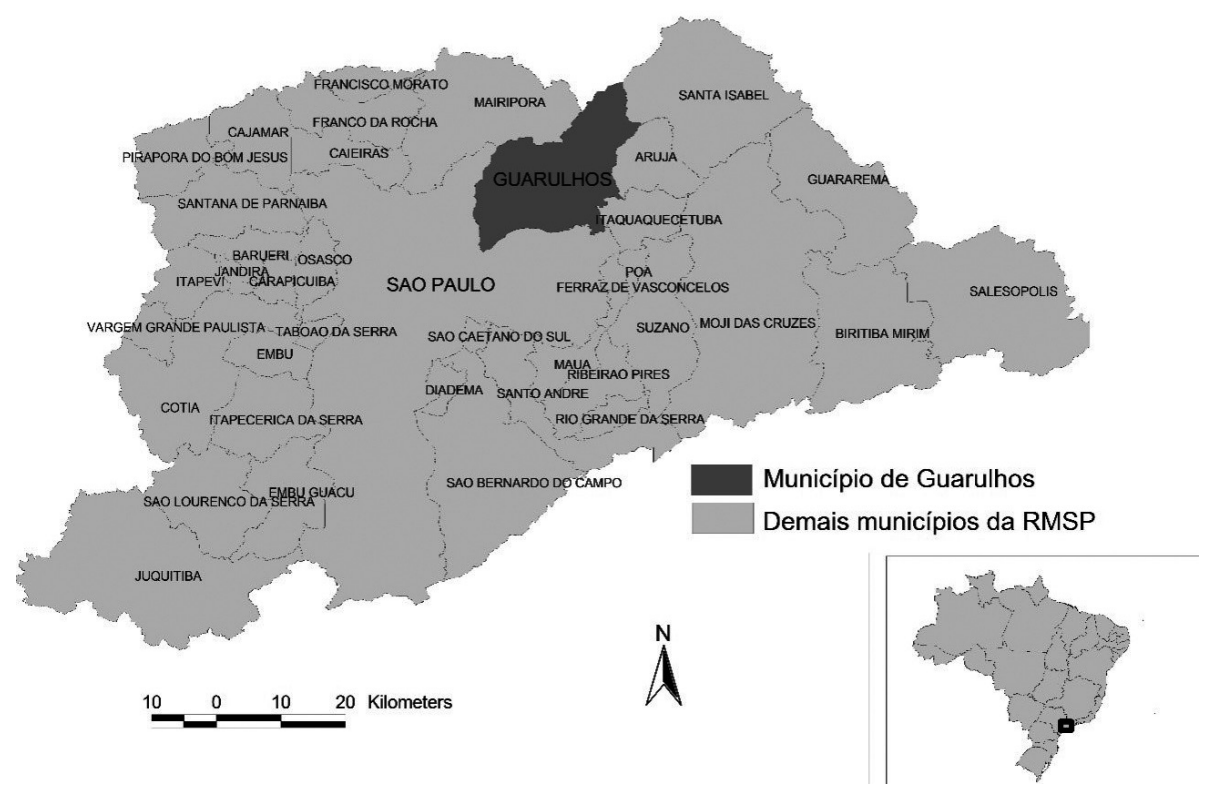

Fonte: IBGE (2010b).

O município de Guarulhos está localizado na província do Planalto Atlântico, com níveis altimétricos acima dos 700 metros acima do nível do mar. A bacia hidrográfica do Alto Tietê está encravada nessa província geomorfológica, com muitas áreas de alta suscetibilidade a processos de inundação. Na Região 
Metropolitana de São Paulo, e no município de Guarulhos em particular, as inundações - episódios que já eram observados devido às condições hidrográficas, climáticas e geomorfológicas locais - tornam-se um grave problema urbano, que se sobrepõe à vulnerabilidade social da população metropolitana e guarulhense. Nesse contexto, a cumulatividade e sobreposição espacial da vulnerabilidade social da população e dos riscos associados às inundações são explicitadas quando ocorrem eventos de grandes proporções, como a grande enchente recém-ocorrida na Metrópole de São Paulo em fevereiro de 2020, momento em que os rios Pinheiros e Tietê transbordaram, ruas foram alagadas, moradores ficaram ilhados, escolas suspenderam aulas e transportes e serviços públicos foram interrompidos (ROSS; MOROZ, 2011; HENRIQUE et al., 2020).

No presente artigo, o conceito de vulnerabilidade socioambiental é operacionalizado empiricamente por meio da construção e análise de indicadores socioambientais em escala intraurbana (setores censitários), integrando dados sociodemográficos do Censo 2010 do IBGE (IBGE, 2010a) com dados (cartografias) que representam áreas de risco ambiental. Esta metodologia combina as duas mencionadas dimensões da vulnerabilidade - exposição e suscetibilidade ao risco ambiental -, gerando um "índice de vulnerabilidade socioambiental"1.

A seguir, são descritos os principais procedimentos metodológicos utilizados para fazer a operacionalização do conceito de vulnerabilidade socioambiental. Primeiramente, a dimensão "exposição ao risco ambiental" foi operacionalizada por meio de um indicador que representa a porcentagem do território sobreposta a áreas de risco ambiental, utilizando o setor censitário como unidade de análise. No presente trabalho, são consideradas como de risco ambiental apenas as áreas localizadas às margens de até 50 metros de cursos d'água, as quais estão sujeitas a enchentes e/ou doenças de veiculação hídrica², deixando-se de fora da análise outros tipos de áreas de risco ambiental, como aquelas com risco de deslizamento.

Para construir o indicador de exposição ao risco ambiental, fez-se a sobreposição espacial da cartografia digital das áreas de risco ambiental (localizadas às margens de até 50 metros de cursos d'água) à malha digital dos setores censitários do Censo 2010 do IBGE do município de Guarulhos (IBGE, 2010b), por meio de

1 Algumas definições de vulnerabilidade consideram até três elementos constituintes da vulnerabilidade: exposição, suscetibilidade e capacidade de adaptação. Porém, para operacionalizar a dimensão "capacidade de adaptação" seria necessário realizar uma análise temporal, o que foge do escopo metodológico do presente trabalho.

2 A cartografia da rede hidrográfica do município de Guarulhos foi obtida junto ao DAEE Departamento de Águas e Energia Elétrica do Estado de São Paulo (DAEE, 2010). 
um Sistema de Informação Geográfica $(\mathrm{SIG})^{3}$. Depois, calculou-se o tamanho e o percentual da área de cada setor sobreposta às áreas de risco ambiental, resultando numa variável quantitativa contínua que mede a porcentagem do território do setor censitário constituída por áreas de risco ambiental (sujeitas a enchentes e/ou de doenças de veiculação hídrica). Por fim, esta variável contínua foi convertida para uma variável categórica ordinal formada por duas categorias (dois grupos), utilizando-se o seguinte critério: 1) setores com mais de 50\% do seu território composto de áreas de risco ambiental foram classificados como de alta exposição ao risco ambiental; e 2) setores com menos de 50\% do seu território composto de áreas de risco ambiental foram classificados como de baixa exposição ao risco ambiental (Figura 2) (ALVES, 2013).

Figura 2 - Sobreposição espacial da cartografia digital das áreas de risco ambiental (margens de 50 metros de cursos d'água) à malha digital dos setores censitários do Censo 2010 do IBGE do município de Guarulhos

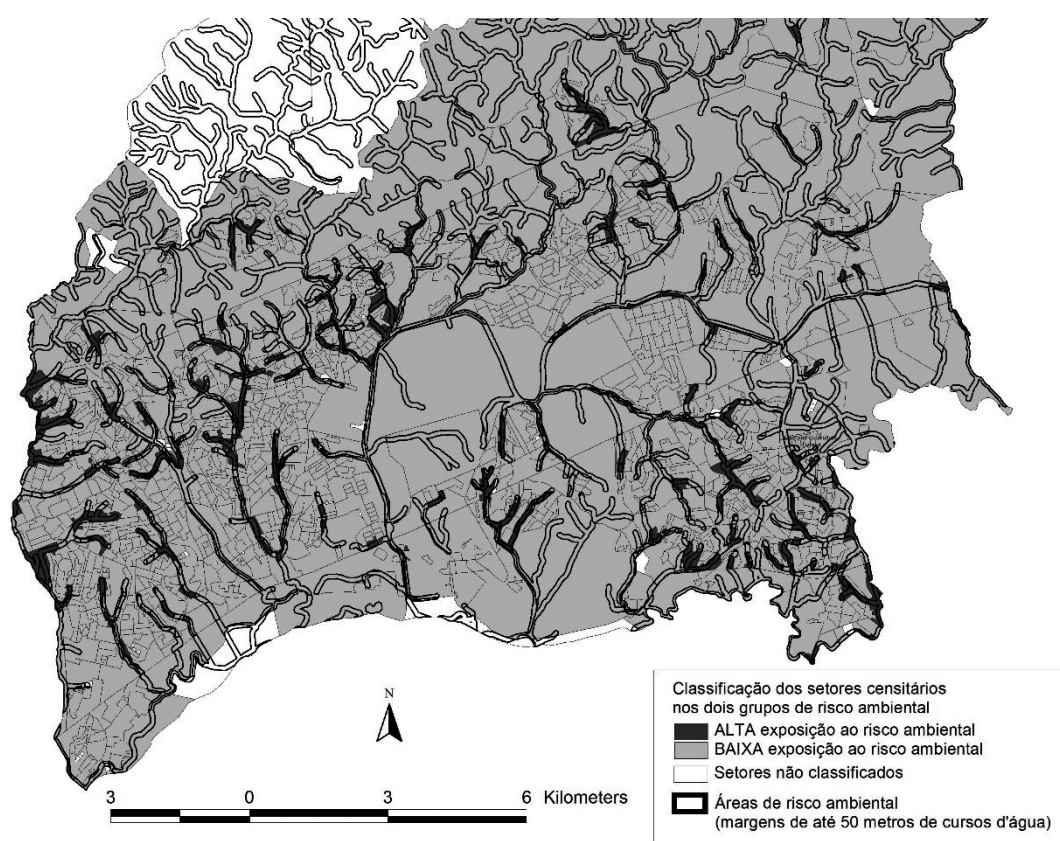

Fontes: DAEE (2010) e IBGE (2010b).

Nota: Para possibilitar uma melhor visualização, em escala intraurbana, da sobreposição espacial das áreas de risco ambiental à malha digital dos setores censitários, foi excluída do mapa a porção do extremo norte do município de Guarulhos, que é a região de domínio da Serra da Cantareira, constituída por áreas rurais, com baixas densidades populacionais e correspondendo a uma proporção mínima da população municipal.

\footnotetext{
Para construção do indicador de exposição ao risco ambiental, utilizou-se o Terra View, que é um software livre, desenvolvido pelo Instituto Nacional de Pesquisas Espaciais (INPE).
} 
Já para operacionalizar a outra dimensão da vulnerabilidade "suscetibilidade ao risco" -, utilizou-se um indicador de renda domiciliar média per capita, que foi obtido nos resultados do Universo do Censo Demográfico de 2010 (IBGE, 2010a), e depois convertido para uma variável categórica ordinal formada por duas categorias, de modo a classificar os setores censitários em dois grupos: 1) setores com renda domiciliar média per capita abaixo de 1 salário mínimo foram classificados como de alta pobreza/suscetibilidade; e 2) setores com renda domiciliar média per capita acima de 1 salário mínimo ${ }^{4}$ foram classificados como de baixa pobreza/suscetibilidade ${ }^{5}$ (ALVES, 2013).

Fazendo uma combinação entre as duas dimensões - exposição e suscetibilidade/pobreza ao risco ambiental -, foi gerado o "índice de vulnerabilidade socioambiental", que consiste em uma variável categórica ordinal com quatro categorias/grupos, descritos no Quadro 1.

Quadro 1 - Construção do índice de vulnerabilidade socioambiental, por meio da combinação das dimensões suscetibilidade/pobreza e exposição ao risco ambiental

\begin{tabular}{|c|c|c|}
\hline \multicolumn{2}{|c|}{ Dimensões } & \multirow{2}{*}{$\begin{array}{c}\text { Índice de } \\
\text { Vulnerabilidade } \\
\text { Socioambiental }\end{array}$} \\
\hline $\begin{array}{c}\text { Exposição ao } \\
\text { risco ambiental }\end{array}$ & $\begin{array}{c}\text { Suscetibilidade/ } \\
\text { Pobreza }\end{array}$ & \\
\hline $\begin{array}{c}\text { ALTA exposição: } \\
\text { Acima de } \mathbf{5 0 \%} \text { de áreas de } \\
\text { risco ambiental }\end{array}$ & $\begin{array}{l}\text { ALTA pobreza/suscetibilidade: } \\
\text { Renda domiciliar per capita } \\
\text { abaixo de } 1 \text { salário mínimo }\end{array}$ & $\begin{array}{c}\text { ALTA } \\
(\text { Grupo 4) }\end{array}$ \\
\hline $\begin{array}{c}\text { BAIXA exposição: } \\
\text { Abaixo de } \mathbf{5 0} \% \text { de áreas de } \\
\text { risco ambiental }\end{array}$ & $\begin{array}{c}\text { ALTA pobreza/suscetibilidade: } \\
\text { Renda domiciliar per capita } \\
\text { abaixo de } 1 \text { salário mínimo }\end{array}$ & $\begin{array}{c}\text { MODERADA } \\
\begin{array}{c}\text { (com alta pobreza) } \\
\text { (Grupo 3) }\end{array} \\
\end{array}$ \\
\hline $\begin{array}{c}\text { ALTA exposição: } \\
\underline{\text { Acima de } \mathbf{5 0 \%} \text { de áreas de }} \\
\text { risco ambiental }\end{array}$ & $\begin{array}{c}\text { BAIXA pobreza/ } \\
\text { suscetibilidade: Renda } \\
\text { domiciliar per capita acima de } \\
1 \text { salário mínimo }\end{array}$ & $\begin{array}{l}\text { MODERADA } \\
\text { (com alto risco) } \\
\quad(\text { Grupo } 2)\end{array}$ \\
\hline $\begin{array}{c}\text { BAIXA exposição: } \\
\text { Abaixo de } \mathbf{5 0} \% \text { de áreas de } \\
\text { risco ambiental }\end{array}$ & $\begin{array}{c}\text { BAIXA pobreza/ } \\
\text { suscetibilidade: Renda } \\
\text { domiciliar per capita acima de } \\
1 \text { salário mínimo }\end{array}$ & $\begin{array}{c}\text { BAIXA } \\
\text { (Grupo 1) }\end{array}$ \\
\hline
\end{tabular}

Fontes: DAEE (2010) e IBGE (2010a).

\footnotetext{
Em 2010, o valor do salário mínimo era de 510 reais.

5 Utiliza-se a noção de pobreza como proxy de suscetibilidade, usando a renda domiciliar média per capita do setor censitário como indicador, devido à renda ser praticamente a única variável socioeconômica disponível nos resultados do Universo do Censo 2010, já que se utiliza o setor como unidade de análise.
} 
Entre as principais limitações metodológicas apontadas em relação ao presente índice de vulnerabilidade socioambiental, destacam-se: 1) o foco exclusivo no risco de enchentes e doenças de veiculação hídrica, deixando-se de fora outros tipos de risco ambiental como deslizamentos; 2) a utilização da renda per capita como proxy de pobreza e de suscetibilidade; e 3) a não incorporação da dimensão "capacidade de adaptação" na operacionalização do conceito de vulnerabilidade.

\section{RESULTADOS E DISCUSSÃO: ANÁLISE COMPARATIVA ENTRE OS QUATRO GRUPOS DE VULNERABILIDADE SOCIOAMBIENTAL}

No presente item são apresentados os resultados da operacionalização empírica do conceito de vulnerabilidade socioambiental, por meio da análise do índice de vulnerabilidade socioambiental. Para isso, é feita a seguir uma análise comparativa entre os quatro grupos de vulnerabilidade socioambiental (representados na Figura 3), em relação a indicadores socioeconômicos e demográficos, selecionados dos resultados do Universo do Censo Demográfico 2010 e apresentados na Tabela 1.

Como mostra a Tabela 1, a população de cerca de 1,2 milhões de habitantes do município de Guarulhos, no ano de 2010, distribui-se da seguinte maneira entre os quatro grupos de vulnerabilidade socioambiental. Nas áreas (setores censitários) com baixa vulnerabilidade socioambiental (Grupo 1) residem 561 mil pessoas, que correspondem a 46,2\% da população do município de Guarulhos no ano 2010. Já nas áreas de moderada vulnerabilidade socioambiental [com alto risco] (Grupo 2) vivem 37 mil pessoas (apenas 3,1\% da população municipal). Nas áreas de moderada vulnerabilidade socioambiental [com alta pobreza] (Grupo 3) residem 497 mil pessoas (40,9\% da população do município). Por fim, nas áreas de alta vulnerabilidade socioambiental (Grupo 4) vivem 119 mil pessoas, que correspondem a 9,8\% da população de Guarulhos.

Comparando-se os níveis de cobertura de saneamento básico dos quatro grupos, pode-se observar que a coleta de lixo está praticamente universalizada no município de Guarulhos, sendo que os quatro grupos possuem quase $100 \%$ dos seus domicílios com lixo coletado. Com relação ao abastecimento de água, enquanto os dois grupos de baixa pobreza (grupos 1 e 2) possuem perto de 100\% dos seus domicílios com acesso à rede geral de água, nos dois grupos de alta pobreza (grupos 3 e 4) o acesso à rede de água é de 96,4\% dos domicílios no Grupo 3 e de 95\% no Grupo 4. 
Figura 3 - Classificação dos setores censitários do município de Guarulhos nos quatro grupos de vulnerabilidade socioambiental

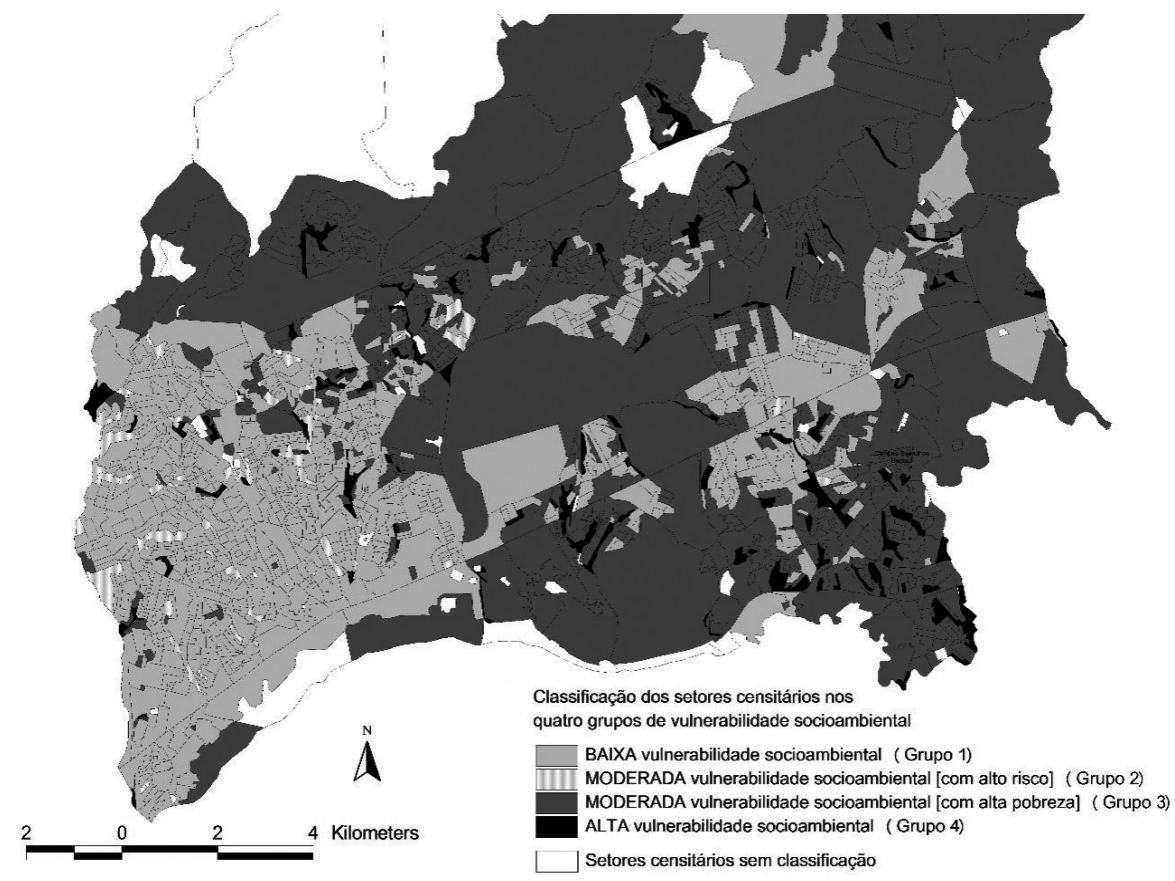

Fontes: DAEE (2010) e IBGE (2010b).

Nota: Para possibilitar uma melhor visualização, em escala intraurbana, da classificação dos setores censitários do município de Guarulhos nos quatro grupos de vulnerabilidade socioambiental, foi excluída do mapa a porção do extremo norte do município de Guarulhos, que é a região de domínio da Serra da Cantareira, constituída por áreas rurais, com baixas densidades populacionais e correspondendo a uma proporção mínima da população municipal.

No que tange a cobertura de esgoto, observam-se diferenças consideráveis entre os quatro grupos. Enquanto no grupo de baixa vulnerabilidade socioambiental 96,7\% dos domicílios estão ligados à rede geral de esgoto, nas áreas de alta vulnerabilidade socioambiental (Grupo 4) esta porcentagem é de apenas $51,6 \%$, revelando uma expressiva carência de cobertura da rede de esgoto para quase metade dos domicílios deste grupo. Já no Grupo 3 (baixo risco ambiental e alta pobreza), o percentual de domicílios ligados à rede geral de esgoto é de $82,5 \%$, bem maior do que o grupo de alta vulnerabilidade socioambiental, o que mostra que a ausência de esgotamento sanitário se manifesta principalmente nas 
residências localizadas em áreas onde há sobreposição espacial entre pobreza e exposição ao risco ambiental de enchentes ${ }^{6}$ (Tabela 1).

Se forem analisadas as características de entorno dos domicílios, também se observam importantes diferenças entre os quatro grupos, particularmente do grupo de alta vulnerabilidade socioambiental em relação aos demais. Como mostra a Tabela 1, nas cinco variáveis referentes ao entorno dos domić́lios (falta de iluminação pública, ruas sem pavimentação, ruas sem arborização, esgoto a céu aberto e lixo nos logradouros), o Grupo 4 apresenta percentuais significativamente piores do que os outros grupos, até mesmo em relação ao Grupo 3, com destaque para ruas sem arborização (34,5\% dos domicílios) e sem pavimentação (11\% dos domicílios), esgoto a céu aberto (5,3\% dos domicílios) e presença de lixo nos logradouros (8,7\% dos domicílios).

No que concerne à cor da pele, a proporção de pessoas de cor preta ou parda é significativamente mais alta nos dois grupos de alta pobreza (grupos 3 e 4), chegando a quase 60\% no conjunto de setores censitários com alta vulnerabilidade socioambiental. No grupo de baixa vulnerabilidade socioambiental, esta proporção é de 33\% (Tabela 1).

Os quatro grupos de vulnerabilidade socioambiental também possuem grandes diferenças em relação aos indicadores de renda. O percentual de domicílios com renda per capita abaixo da linha da pobreza (abaixo de um quarto salário mínimo, inclusive os sem renda $)^{7}$ nas áreas de alta vulnerabilidade socioambiental $(9,9 \%)$ é sensivelmente maior do que nos domicílios do Grupo 3 (7,5\%), que também compartilham a dimensão pobreza, mas não estão expostos ao risco ambiental. Já nos grupos de baixa pobreza (1 e 2), são bem menores os percentuais de domicílios abaixo da linha da pobreza, com 2,2\% nas áreas do Grupo 1 (com baixo risco ambiental) e 2,7\% nas do Grupo 2 (com alto risco ambiental).

Tomando-se como referência o percentual de domicílios com renda per capita abaixo de meio salário mínimo, as diferenças entre os quatro grupos são ainda maiores, com 34,7\% dos domicílios abaixo deste patamar nas áreas classificadas como de alta vulnerabilidade socioambiental, contrastando com apenas 10,3\% nas áreas de baixa vulnerabilidade socioambiental, e revelando

\footnotetext{
O Grupo 2 (alto risco ambiental e baixa pobreza) possui 90,2\% dos seus moradores em domicílios ligados à rede geral de esgoto.

Esta linha de pobreza tem sido comumente adotada como referência no Brasil. Ver Rocha (2003).
} 
novamente a sobreposição de situações de pobreza e exposição ao risco ambiental nos setores do Grupo $4^{8}$.

Os resultados também revelam uma expressiva variação da renda domiciliar média per capita entre os grupos de vulnerabilidade socioambiental. Se nas áreas de alta vulnerabilidade socioambiental, a renda domiciliar per capita média é de apenas 342 reais ( 0,7 salários mínimos), nas áreas de baixa vulnerabilidade socioambiental atinge 907 reais (1,8 salários mínimos em 2010) ${ }^{910}$ (Tabela 1).

A proporção de pessoas residentes em aglomerados subnormais (áreas com características de favela segundo classificação do IBGE) é uma variável que expressa a associação entre pobreza e falta de infraestrutura urbana, e que também pode revelar situações de exposição ao risco ambiental (TASCHNER, 2000; ALVES, 2013). Nas áreas classificadas como de alta vulnerabilidade socioambiental, nada menos do que $69,7 \%$ da população vive em aglomerados subnormais, ou seja, em áreas classificadas como favelas. Já nas áreas que também compartilham da dimensão pobreza, mas que não são expostas ao risco ambiental (classificadas no Grupo 3), a porcentagem de população residente em aglomerados subnormais é bem mais baixa (26,6\%). Nas áreas de baixa pobreza (grupos 1 e 2), praticamente não há população residente em aglomerados subnormais $^{11}$ (Tabela 1).

Essa grande diferença no percentual de população favelada entre os dois grupos de alta pobreza (grupos 3 e 4) mostra que a presença de favelas não está associada apenas à pobreza, mas sim à coincidência espacial entre pobreza e exposição a risco ambiental, o que revela a fortíssima concentração e sobreposição de problemas e riscos sociais e ambientais em determinadas áreas do município de Guarulhos, como os setores censitários classificados como de alta vulnerabilidade socioambiental.

\footnotetext{
8 Nos grupos intermediários, os percentuais de domicílios com renda per capita abaixo de $1 / 2$ salário mínimo são de $12 \%$ nas áreas do Grupo 2 (alto risco ambiental e baixa pobreza) e 28,5\% nas áreas do Grupo 3 (baixo risco ambiental e alta pobreza).

$9 \quad$ Nos grupos intermediários, a renda domiciliar per capita média é de 833 reais (1,6 salários mínimos) nas áreas do Grupo 2 (alto risco ambiental e baixa pobreza) e de 388 reais $(0,8$ salários mínimos) nas áreas do Grupo 3 (baixo risco ambiental e alta pobreza).

10 Em 2010, o valor do salário mínimo era de 510 reais.

11 Com percentuais de pessoas residentes em aglomerados subnormais de meros $0,1 \%$ nas áreas do Grupo 1 (com baixo risco ambiental) e zero nas áreas do Grupo 2 (com alto risco ambiental).
} 
Tabela 1 - Indicadores socioeconômicos e demográficos por categoria de vulnerabilidade socioambiental. Município de Guarulhos, 2010

\begin{tabular}{|c|c|c|c|c|}
\hline \multirow[b]{2}{*}{$\begin{array}{l}\text { Indicadores } \\
\text { socioeconômicos e } \\
\text { demográficos }\end{array}$} & \multicolumn{4}{|c|}{ Vulnerabilidade Socioambiental } \\
\hline & $\begin{array}{c}\text { ALTA } \\
\text { (Grupo 4) }\end{array}$ & $\begin{array}{l}\text { MODERADA } \\
\text { com alta } \\
\text { pobreza } \\
\text { (Grupo 3) }\end{array}$ & $\begin{array}{l}\text { MODERADA } \\
\text { com alto } \underline{\text { risco }} \\
(\text { Grupo } 2)\end{array}$ & $\begin{array}{c}\text { BAIXA } \\
\text { (Grupo 1) }\end{array}$ \\
\hline População residente & 118.819 & 496.873 & 37.505 & 561.446 \\
\hline Número de domicílios & 32.513 & 140.199 & 11.821 & 176.906 \\
\hline $\begin{array}{l}\text { Distribuição da } \\
\text { população residente (\%) }\end{array}$ & 9,78 & 40,91 & 3,09 & 46,22 \\
\hline $\begin{array}{l}\text { Distribuição do total de } \\
\text { domicílios }(\%)\end{array}$ & 9,00 & 38,79 & 3,27 & 48,94 \\
\hline $\begin{array}{l}\text { Domicílios com coleta de } \\
\text { lixo }(\%)\end{array}$ & 99,09 & 99,41 & 99,97 & 99,92 \\
\hline $\begin{array}{l}\text { Domicílios com rede } \\
\text { geral de água }(\%)\end{array}$ & 94,97 & 96,43 & 99,80 & 98,87 \\
\hline $\begin{array}{l}\text { Domicílios c/ rede geral } \\
\text { de esgoto }(\%)\end{array}$ & 51,58 & 82,46 & 90,17 & 96,70 \\
\hline $\begin{array}{l}\text { Domicílios sem } \\
\text { iluminação pública (\%) }\end{array}$ & 3,14 & 4,42 & 1,00 & 0,79 \\
\hline $\begin{array}{l}\text { Domicílios em rua s/ } \\
\text { pavimentação }(\%)\end{array}$ & 10,98 & 10,57 & 1,50 & 1,21 \\
\hline $\begin{array}{l}\text { Domicílios em rua sem } \\
\text { arborização }(\%)\end{array}$ & 34,53 & 30,85 & 27,75 & 24,68 \\
\hline $\begin{array}{l}\text { Domicílios c/ esgoto a } \\
\text { céu aberto }(\%)\end{array}$ & 5,34 & 3,62 & 2,00 & 1,44 \\
\hline $\begin{array}{l}\text { Domicílios c/ lixo nos } \\
\text { logradouros }(\%)\end{array}$ & 8,73 & 5,31 & 1,01 & 1,95 \\
\hline Pessoas de cor branca $(\%)$ & 39,26 & 43,76 & 61,68 & 64,66 \\
\hline $\begin{array}{l}\text { Pessoas de cor preta ou } \\
\text { parda }(\%)\end{array}$ & 59,41 & 55,05 & 37,10 & 33,29 \\
\hline $\begin{array}{l}\text { Domicílios com renda } \\
\text { per capita de até } 1 / 4 \text { salário } \\
\text { mínimo }(\%)\end{array}$ & 9,88 & 7,49 & 2,66 & 2,15 \\
\hline $\begin{array}{l}\text { Domicílios com renda } \\
\text { per capita de até } 1 / 2 \text { salário } \\
\text { mínimo }(\%)\end{array}$ & 34,68 & 28,51 & 12,01 & 10,34 \\
\hline $\begin{array}{l}\text { Renda per capita média } \\
\text { (reais) }\end{array}$ & 342 & 388 & 833 & 907 \\
\hline $\begin{array}{l}\text { Renda per capita média } \\
\text { (sal. min.) }\end{array}$ & 0,67 & 0,76 & 1,63 & 1,78 \\
\hline $\begin{array}{l}\text { População em } \\
\text { aglomerados subnormais }\end{array}$ & 82.816 & 132.261 & 0 & 725 \\
\hline $\begin{array}{l}\text { População aglomerados } \\
\text { subnormais }(\%)\end{array}$ & 69,70 & 26,62 & 0,00 & 0,13 \\
\hline
\end{tabular}

Fontes: DAEE (2010) e IBGE (2010a, 2010b). 
Sintetizando os resultados, pode-se constatar que as áreas (setores censitários) com alta vulnerabilidade socioambiental possuem características socioeconômicas significativamente piores do que aquelas com baixa vulnerabilidade socioambiental, e mesmo em relação às situações intermediárias de moderada vulnerabilidade socioambiental (grupos 2 e 3). Entre as variáveis que mais diferenciam as áreas de alta vulnerabilidade socioambiental em relação aos outros três grupos, destacam-se a cobertura de esgoto, as características de entorno dos domicílios (ruas sem arborização e sem pavimentação, lixo nos logradouros e esgoto a céu aberto), e principalmente a população residente em aglomerados subnormais. Além disso, praticamente todos os indicadores socioeconômicos das áreas de alta vulnerabilidade socioambiental (Grupo 4) são piores do que os das áreas do Grupo 3, as quais também possuem alta pobreza, mas não estão expostas ao risco ambiental.

Deste modo, os resultados revelam que a exposição ao risco ambiental possui uma forte associação e sobreposição espacial com a pobreza, gerando situações de alta vulnerabilidade socioambiental. O principal fator que explica isso é a grande concentração de aglomerados subnormais (favelas) nas áreas de risco ambiental, que geralmente correspondem a áreas não edificantes, consideradas inadequadas pelas legislações urbanística e ambiental para ocupação urbana, seja porque oferecem risco ambiental, seja porque são áreas de preservação permanente. Geralmente são áreas (públicas ou privadas) invadidas por assentamentos precários, que se configuram como áreas de favela. Outra possível explicação é que essas áreas de alta vulnerabilidade socioambiental muitas vezes são as localidades mais acessíveis à população de mais baixa renda, pois são áreas muito desvalorizadas no mercado de terras por serem pouco propícias à ocupação, devido às características de risco e falta de infraestrutura urbana (ALVES, 2006, 2013).

\section{CONSIDERAÇÕES FINAIS}

No presente artigo, o conceito de vulnerabilidade socioambiental foi operacionalizado empiricamente, por meio da construção de um índice que faz a integração de indicadores sociodemográficos do Censo 2010 do IBGE com cartografias que representam áreas de risco ambiental, de modo a fazer uma análise de situações de vulnerabilidade socioambiental em escala intraurbana no município de Guarulhos-SP.

A análise dos dados mostra que determinadas áreas, espalhadas pelo território do município, possuem uma forte concentração e sobreposição espacial 
de situações de exposição a risco ambiental e de situações de suscetibilidade/ pobreza, produzindo situações de alta vulnerabilidade socioambiental. Os resultados mostram ainda que 119 mil pessoas vivem nessas áreas classificadas como de alta vulnerabilidade socioambiental, correspondendo a quase $10 \% \mathrm{da}$ população de Guarulhos, o que são números expressivos e preocupantes, em vista dos cenários de aumento da intensidade e frequência de eventos climáticos extremos e dos riscos ambientais associados, nos próximos anos e décadas, no contexto das mudanças climáticas (NOBRE; YOUNG, 2011).

Nesse sentido, subsídios poderão ser fornecidos, por meio dos resultados deste trabalho, para que o município de Guarulhos possa planejar políticas públicas de mitigação das situações de vulnerabilidade socioambiental e de adaptação às mudanças climáticas, nos próximos anos e décadas. Entre essas políticas públicas, destacam-se as de habitação e saneamento, especialmente políticas de urbanização de favelas, que poderiam ser muito eficazes para a diminuição das situações de alta vulnerabilidade socioambiental no município, tendo em vista que a maioria das áreas nesta situação é de favelas (ALVES, 2006, 2013).

Além disso, ao fazer a integração de fontes censitárias de dados sociodemográficos com cartografias ambientais (áreas de risco), o trabalho procura trazer uma contribuição metodológica para os estudos urbanos e ambientais, em particular para as análises de situações de vulnerabilidade socioambiental em escala intraurbana. Estas metodologias e indicadores podem ser replicados e adaptados para outras áreas urbanas e metropolitanas do Brasil, uma vez que utilizam a malha digital de setores censitários do Censo Demográfico de 2010 do IBGE (ALVES, 2009, 2013).

No entanto, é importante ressalvar que o presente índice de vulnerabilidade socioambiental possui limitações, como a não incorporação de outros tipos de risco ambiental, principalmente as áreas com risco de deslizamento. Outra lacuna é a não incorporação da dimensão "capacidade de adaptação" na operacionalização do conceito de vulnerabilidade. Nesse sentido, a seleção e incorporação de outros indicadores e variáveis para compor o referido índice certamente afetaria os seus resultados e aprimoraria a representação que foi feita da vulnerabilidade socioambiental.

A literatura traz diversos estudos com índices compostos que incorporam grande quantidade e variedade de indicadores e dimensões da vulnerabilidade (SAKAI et al., 2017; DOWNING et al., 2001). Porém, muitos desses índices também possuem limites e problemas analíticos e metodológicos, como, por exemplo, a dificuldade de representar situações de vulnerabilidade em escalas espaciais mais detalhadas. Portanto, a despeito das limitações metodológicas 
apontadas, o índice de vulnerabilidade socioambiental do presente artigo pode ser considerado um indicador exigente, que representa bem duas importantes dimensões da vulnerabilidade socioambiental, em uma escala espacial intraurbana.

\section{REFERÊNCIAS}

ADGER, W. N. Vulnerability. Global Environmental Change, [S. l.], v. 16, n. 3, p. 268- 281, 2006.

ALVES, H. P. F. Vulnerabilidade socioambiental na metrópole paulistana: uma análise sociodemográfica das situações de sobreposição espacial de problemas e riscos sociais e ambientais. Revista Brasileira de Estudos de População, [ $S$. l.], v. 23, n. 1, p. 43-59, jan./jun. 2006.

ALVES, H. P. F. Metodologias de integração de dados sociodemográficos e ambientais para análise da vulnerabilidade socioambiental em áreas urbanas no contexto das mudanças climáticas. In: HOGAN, D.; MARANDOLA JÚNIOR, E. (org.). População e mudança climática: dimensões humanas das mudanças ambientais globais. Campinas: Núcleo de Estudos de População - Nepo/ Unicamp; Brasília: UNFPA, 2009. p. 75-105.

ALVES, H. P. F. Análise da vulnerabilidade socioambiental em Cubatão - SP por meio da integração de dados sociodemográficos e ambientais em escala intraurbana. Revista Brasileira de Estudos de População, [S. l.], v. 30, n. 2, p. 349-366, jul./dez. 2013.

ALVES, H. P. F.; TORRES, H. G. Vulnerabilidade socioambiental na cidade de São Paulo: uma análise de famílias e domicílios em situação de pobreza e risco ambiental. São Paulo em Perspectiva, São Paulo, v. 20, n. 1, p. 44-60, jan./mar. 2006.

ALVES, H. P. F.; ALVES, C. D.; PEREIRA, M. N.; MONTEIRO, A. M. V. Dinâmicas de urbanização na hiperperiferia da metrópole de São Paulo: análise dos processos de expansão urbana e das situações de vulnerabilidade socioambiental em escala intraurbana. Revista Brasileira de Estudos de População, [S. l.], v. 27, p. 141-159, jan./jun. 2010.

BALICA, S. F.; DOUBEN, N.; WRIGHT, N. G. Flood vulnerability indices at varying spatial scales. Water Science and technology, [S. l.], v. 60, n. 10, p. 2571-2580, 2009. 
BONDUKI, N.; ROLNIK, R. Periferia da Grande São Paulo: reprodução do espaço como expediente de reprodução da força de trabalho. In: MARICATO, E. (org.). A produção capitalista da casa (e da cidade) do Brasil industrial. São Paulo: Alfa-Ômega, 1982. p. 117-154.

BRAGA, T. M.; OLIVEIRA, E. L.; GIVISIEZ, G. H. N. Avaliação de metodologias de mensuração de risco e vulnerabilidade social a desastres naturais associados à mudança climática. São Paulo em Perspectiva, São Paulo, v. 20, n. 1, p. 81-95, jan./mar. 2006.

CARMO, R.; VALENCIO, N. Segurança humana no contexto dos desastres. São Carlos, SP: RiMa Editora, 2014.

CHAMBERS, R. Vulnerability, coping and policy. IDS Bulletin, [S. l.], v. 20, n. 2, p. 1-7, 1989 .

CIDADE, L. C. F. Urbanização, ambiente, risco e vulnerabilidade: em busca de uma construção interdisciplinar. Cadernos Metrópole, São Paulo, v. 15, n. 29, p. 171-191, jan./jun. 2013.

CUTTER S. L. (org.) Environmental risks and hazards. London: PrenticeHall, 1994.

CUTTER S. L. Vulnerability to environmental hazards. Progress in Human Geography, [S. l.], v. 20, n. 4, p. 529-539, Dec. 1996.

DAEE. Cartografia da rede hidrográfica da Região Metropolitana de São Paulo. São Paulo: DAEE, 2010.

DE SHERBININ, A.; SCHILLER, A.; PULSIPHER, A. The vulnerability of global cities to climate hazards. Environment \& Urbanization Journal, London, v. 19, n. 1, p. 39-64, Apr. 2007.

DOWNING, T. E.; BUTTERFIELD, R.; COHEN, S.; HUQ, S.; MOSS, R.; RAHMAN, A.; SOKONA, Y.; STEPHEN, L. Vulnerability Indices: climate change impacts and adaptation. Nairobi: UNEP, 2001.

GALLOPÍN, G. C. Linkages between vulnerability, resilience, and adaptive capacity. Global Environmental change, [S. l.], v. 16, n. 3, p. 293-303, 2006.

GRAIZBORD, B. Planning for adaptation in a megacity: a case study of the Mexico City Metropolitan Area. In: MARTINE, G.; SCHENSUL, D. (ed.). The demography of adaptation to climate change. New York, London and Mexico City: UNFPA: IIED: El Colegio de México, 2013. p. 158-176. 
HENRIQUE, A. et al. Temporal provoca alagamentos, interdições e caos no transporte em São Paulo. Folha de S. Paulo, São Paulo, ano 99, n. 33.186, 11 fev. 2020. Cotidiano, p. C1.

HUQ S.; KOVATS S.; REID H.; SAT'TERTHWAITE D. Editorial: Reducing risks to cities from disasters and climate change. Environment \& Urbanization Journal, London, v. 19, n.1, p. 3-15, Apr. 2007.

IBGE. Censo demográfico 2010. IBGE, Rio de Janeiro, 2010a. Disponível em: http://censo2010.ibge.gov.br. Acesso em: 08 out. 2019.

IBGE. Malhas digitais. IBGE, Rio de Janeiro, 2010b. Disponível em: https:// mapas.ibge.gov.br/bases-e-referenciais/bases-cartograficas/malhas-digitais. Acesso em: 08 out. 2019.

IPCC. IPCC Fourth Assessment Report (Working Group II). Cambridge, UK: Cambridge UniversityPress, 2007.

KAZTMAN, R.; BECCARIA, L.; FILGUEIRA, F.; GOLBERT, L.; KESSLER, G. Vulnerabilidad, activos y exclusión social en Argentina y Uruguay. Santiago de Chile: OIT, 1999.

LINDOSO, D. P. Vulnerabilidade e Resiliência: potenciais, convergências e limitações na pesquisa interdisciplinar. Ambiente \& Sociedade, São Paulo v. XX, n. 4, p. 131-148, out./dez. 2017.

MARANDOLA, E.; HOGAN, D. J. Natural hazards: o estudo geográfico dos riscos e perigos. Ambiente \& Sociedade, São Paulo, n. 2, p. 95-109, 2004.

MARCONDES, M. J. A. Cidade e natureza: proteção dos mananciais e exclusão social. São Paulo: Studio Nobel; EDUSP; FAPESP, 1999. (Coleção Diade Aberta).

MARICATO, E. Metrópole na periferia do capitalismo: ilegalidade, desigualdade e violência. São Paulo: Hucitec, 1996.

MARICATO, E. Metrópole, legislação e desigualdade. Estudos Avançados, São Paulo, v. 17, n. 48, p. 151-167, ago. 2003.

MARTINE, G.; OJIMA, R. The challenges of adaptation in an early but unassisted urban transition. In: MARTINE, G.; SCHENSUL, D. (ed.). The demography of adaptation to climate change. New York, London and Mexico City: UNFPA, IIED and El Colegio de México, 2013. p. 138-157.

MARTINE, G.; SCHENSUL, D. (ed.). The demography of adaptation to climate change. New York, London and Mexico City: UNFPA, IIED and El Colegio de México, 2013. 
MILANEZ, B.; FONSECA, I. F. O discurso da justiça climática no contexto brasileiro: possibilidades e perspectivas. In: MOTTA R. S., HARGRAVE J., LUEDEMANN G., GUTIERREZ M. B. S. (ed.). Mudança do clima no Brasil: aspectos econômicos, sociais e regulatórios. Brasília: IPEA, 2011. p. 261-276.

MOSER, C. The asset vulnerability framework: reassessing urban poverty reduction strategies. World Development, [S. l.], v. 26, n. 1, 1998.

MOSS, R. H. et al. The next generation of scenarios for climate change research and assessment. Nature, [S. l.], v. 463, n. 7282, p. 747-756, 2010.

NOBRE, C. A.; YOUNG, A. F. (ed.). Vulnerabilidades das megacidades brasileiras às mudanças climáticas: Região Metropolitana de São Paulo. Relatório Final. São Paulo: CCST/INPE; NEPO/UNICAMP; FM/USP; IPT, 2011.

O'BRIEN, K.L.; LEICHENKO, R.; KELKARC, U.; VENEMAD, H.; AANDAHL, G.; TOMPKINS, H.; JAVED, A.; BHADWAL, S.; BARG, S.; NYGAARD, L.; WEST, J. Mapping vulnerability to multiple stressors: climate change andglobalization in India. Global Environmental Change, [S. l.], v. 14, n. 4, p. 303-313, 2004.

O'BRIEN, K.L.; ERIKSEN, S.; NYGAARD, L. P.; SCHJOLDEN, A. N. Why different interpretations of vulnerability matter in climate change discourses. Climatepolicy, [S. l.], v. 7, n. 1, p. 73-88, 2007.

OJIMA, R. As dimensões demográficas das mudanças climáticas: cenários de mudança do clima e as tendências do crescimento populacional. Revista Brasileira de Estudos de População, [S. l.], v. 28, n. 2, p.389-403, jul./dez. 2011.

OJIMA, R., HOGAN, D. J. Mobility, urban sprawl and environmental risks in Brazilian urban agglomerations: challenges for the urban sustainability in a developing country. In: DE SHERBININ, A.; RAHMAN, A.; BARBIERI, A.; FOTSO, J.C.; ZHU, Y. (ed.). Urban population and environment dynamics in the developing world: case studies and lessons learned. Paris: International Cooperation in National Research in Demography (CICRED), 2009. p. 281-316. REIS, N. G.; TANAKA, M. S. Brasil: estudos sobre dispersão urbana. São Paulo: FAU-USP, 2007.

ROCHA, S. Pobreza no Brasil: afinal, de que se trata? Rio de Janeiro: FGV Editora, 2003.

ROSS, J.; MOROZ, I. Mapa geomorfológico do estado de São Paulo. Revista do Departamento de Geografia, São Paulo, v. 10, p. 41-58, 1996. 
SAKAI, P. et al. Climate change adaptation in Ciudad del Este: starting-point vulnerability assessment. Paraquaria Natural, [S. l.], v. 5, n. 2, p. 19-31, 2017.

SMOLKA, M. Meio ambiente e estrutura urbana. In: MARTINE, G. (org.). População, meio ambiente e desenvolvimento: verdades e contradições. Campinas: Editora da Unicamp, 1993. p. 133-147.

TASCHNER, S. P. Degradação ambiental em favelas de São Paulo. In: TORRES, H.; COSTA, H. (org.). População e meio ambiente: debates e desafios. São Paulo: Editora Senac, 2000. p. 271-297.

TORRES, H.; MARQUES, E. Reflexões sobre a hiperperiferia: novas e velhas faces da pobreza no entorno metropolitano. Revista Brasileira de Estudos Urbanos e Regionais, São Paulo, n. 4, p. 49-70, 2001.

TORRES, H. G.; ALVES, H. P. F.; OLIVEIRA, M. A. São Paulo peri-urban dynamics: some social causes and environmental consequences. Environment \& Urbanization Journal, London, v. 19, n. 1, p. 207-223, Apr. 2007.

TORRES, H.; MARQUES, E.; FERREIRA, M. P.; BITAR, S. Pobreza e espaço: padrões de segregação em São Paulo. Estudos Avançados, São Paulo, v.17, n. 47, p. 97-128, jan./abr. 2003.

UNFPA. Situação da População Mundial 2007: desencadeando o potencial do crescimento urbano. Fundo de População das Nações Unidas. Nova York: UNFPA, 2007.

VINCENT, K. Creating an index of social vulnerability to climate change for Africa. Tyndall Center for Climate Change Research, [S. l.], n. 56, p. 1-50, 2004. 